Tohoku J. Exp. Med., 2009, 218, 325-329

\title{
Gender Differences in the Susceptibility to Renal Ischemia- Reperfusion Injury in BALB/c Mice
}

\author{
Honglin Hu, ${ }^{1}$ Gongxian Wang, ${ }^{2}$ Frédéric Batteux ${ }^{3}$ and Carole Nicco ${ }^{3}$ \\ ${ }^{1}$ Department of Surgery, The Medical College of Nanchang University, Nanchang, P.R China
${ }^{2}$ Department of Urology, The First Affiliated Hospital of Nanchang University, Nanchang, P.R China
${ }^{3}$ Université Paris Descartes, Faculté de Médecine, Hôpital Cochin, AP-HP, Laboratoire d'immunologie, Paris
cedex 14, France
}

Recent studies have shown the remarkable gender differences in the susceptibility or expression of many diseases. The mechanism underlying the gender differences is unclear. In the present study, we evaluated the effects of gender differences and different ischemia time on the renal ischemia-reperfusion injury (IRI). The IRI was induced in the bilateral kidneys of 156 male and 30 female BALB/c mice. Renal function, serum creatinine and blood urea nitrogen, and pathology of the kidneys were examined at $24 \mathrm{hr}$ after IRI. Renal IRI was generated successfully in 182 of 186 mice with a $97.85 \%$ success rate. The levels of serum creatinine and blood urea nitrogen were significantly increased in male mice subjected to $30 \mathrm{~min}, 35 \mathrm{~min}$, or $45 \mathrm{~min}$ of renal ischemia and in female mice subjected to $75 \mathrm{~min}$ of renal ischemia, compared to the control group at $24 \mathrm{hr}$ after operation. In males following $35 \mathrm{~min}$ or $45 \mathrm{~min}$ of ischemia and in females following 75 min of ischemia, typical acute tubular necrosis was found in the areas of corticomedullary junction and the histopathologic scores, which represent the degree of renal tissue injuries, were significantly increased. In view of our data, the kidneys of male are much more susceptible to IRI than those of female. The optimal ischemia time of kidney is $35-45 \mathrm{~min}$ in males and $75 \mathrm{~min}$ in females for generating a stable model of IRI in mice. Investigation of the gender differences might provide a new area for mechanistic study of renal IRI. ischemia time.

Tohoku J. Exp. Med., 2009, 218 (4), 325-329. C 2009 Tohoku University Medical Press

Renal ischemia-reperfusion injury (IRI) is a complex pathophysiologic process that occurs in the context of cardiac arrest with recovery, transplantation, heminephrectomy and vascular surgery which is a common cause of renal cell death (Gobé et al. 1999), acute renal failure (ARF), delayed graft function (Perico et al. 2004) and renal graft rejection (Schlichting et al. 2006). There is no specific treatment for this devastating clinical syndrome, partially due to the relatively poor understanding of the pathophysiology of this disease. Animal experiments are frequently applied in the kidney IRI research to better understand this disease in human. So it is pivotal to establish an ideal animal model of kidney IRI.

Gender differences characterize the susceptibility or expression of many diseases. Sexual dimorphism in response to renal disease has been noted for many years. There are gender differences in many renal disease models, including age-related glomerular injury (Baylis 1994), hypertension ( $\mathrm{Ji}$ et al. 2005), polycystic kidney disease (Stringer et al. 2005) and diabetic nephropathy (Tomiyoshi et al. 2002). In recent years experimental science is now producing tantalizing clues that gender differences also play a role in the kidney's response to ischemia. Females have significantly improved survival when exposed to profound renal ischemia. Estradiol treatment of males improves survival, but ovariectomy or testosterone administration to females does not (Müller et al. 2002). Park et al found that males had significantly greater functional and histologic renal injury from ischemia (Park et al. 2004). The mechanism underlying the gender differences remains incompletely understood.

The present study has examined and compared the gender differences in renal IRI models in BALB/c mice, moreover, explored the optimal ischemia time of kidney for the males and females. Thus, we established kidney IRI models with micro-artery clamps to clamp bilateral renal arteries in 156 male and 30 female BALB/c mice. Extensive experience has accumulated in our laboratory with kidney IRI models in BALB/c mice. Our findings reveal that the kidneys of males are much more susceptible to IRI than those of females. To achieve a stable model of renal IRI in mice, the optimal renal ischemia time is $35-45$

Received June 4, 2009; revision accepted for publication July 1, 2009. doi:10.1620/tjem.218.325

Correspondence: Gongxian Wang, Department of Urology, the First Affiliated Hospital of Nanchang University, No.17 Yongwai Street,

Nanchang, 330006, P.R China.

e-mail: wanggx-mr@126.com 
min in males and $75 \mathrm{~min}$ in females.

\section{Materials and Methods}

Experimental Animals

Healthy BALB/c mice (156 male mice and 30 female mice), weighing 20-25 g, were used in the experiments. They were housed in macrolon cages under standard laboratory conditions (temperature: $24 \pm 2^{\circ} \mathrm{C}$ and relative humidity: 55\%). The animals were given standard mouse chow and tap water ad libitum. This study was conducted in compliance with approved animal experimental procedures by the Animal Care and Use Committee at Paris Descartes University. Animals were fasted for 8-12 hr before the experiment.

\section{Experimental Design}

According to different kidney ischemia time, the male mice were divided into four groups ( $0 \mathrm{~min}, 30 \mathrm{~min}, 35 \mathrm{~min}$, and $45 \mathrm{~min})$, and the female mice were divided into five groups ( $0 \mathrm{~min}, 30 \mathrm{~min}, 45$ $\min , 60 \mathrm{~min}$, and $75 \mathrm{~min}$ ).

\section{Surgical Procedure}

To induce renal IRI, the mice were anesthetized by intraperitoneal injections of avertin $(200 \mathrm{mg} / \mathrm{Kg})(2,2$ Tribromoethanol $0.04 \mathrm{M}$ 2-Methyl-2-Butanol 2.5\%). A midline incision was made. Then, the renal pedicles were exposed by abdominal incision and clamped bilaterally for different time (30-75 $\mathrm{min})$. For reperfusion, the clamps were released and the kidneys were monitored for color change to confirm blood reflow before suturing the incisions. After suturing, 0.3 $\mathrm{ml}$ saline was given intraperitoneally. For sham control groups, animals were operated similarly without renal pedicle clamping. The surgery was conducted on a thermostatic stage and the body temperature was maintained at $35-37^{\circ} \mathrm{C}$ with a heating pad.

Besides the mice for observing survival rate, animals were sacrificed at $24 \mathrm{hr}$ after reperfusion, kidneys were isolated and quick frozen in liquid nitrogen and stored at $-80^{\circ} \mathrm{C}$ until further analysis.

\section{Standards that estimate the success of kidney IRI}

In the operation, when the bilateral renal pedicles were clamped with an atraumatic vascular clip, the color of kidney was changed into dark red or paleness. After the clamps were removed, the kidneys were observed for restoration of blood flow and returned to their original color. The mice were resuscitated and recovered their normal activities gradually at 1-3 hr after the operation. Scr and BUN were elevated postoperatively.

\section{Serum Biochemical Evaluation}

Blood samples were obtained from orbit angular vein at $24 \mathrm{hr}$ after reperfusion. The blood was centrifuged (4500 rpm for $10 \mathrm{~min}$ ) to separate serum. To monitor renal function, levels of serum creatinine and blood urea nitrogen (BUN) were measured.

\section{Histopathologic Evaluation of Kidney}

For histopathological examination, kidneys were collected, cut coronally, fixed in $10 \%$ formaldehyde, and embedded in paraffin. Five-micrometer sections were prepared and stained with hemotoxylin and eosin (H\&E).

The sections were scored with a semiquantitative scale designed to evaluate changes in the kidney $24 \mathrm{hr}$ after IRI (Rabb et al. 1994). Specifically, one whole deep coronal section was examined under the microscope and graded according to extent of tubular necrosis, based on percentage of involvement of the kidney. Higher scores represented more severe damage (maximum score $=4$ ): $0=$ normal kidney, $1=$ minimal necrosis, $<5 \%$ involvement; $2=$ mild necrosis, $5-25 \%$ involvement; $3=$ moderate necrosis, $25 \%-75 \%$ involvement; and $4=$ severe, $>75 \%$ involvement.

\section{Statistical Analysis}

Statistical analysis was performed with GraphPad Prism 5.0. All data were expressed as mean values \pm S.E. The statistical significance of intergroup differences in the mean values of histopathologic scores and biochemical parameters was evaluated by the Student's $t$ test or one-way ANOVA plus the Tukey post hoc multiple-comparisons test. A $p$ value $<0.05$ is considered significant. Survival curve were analyzed by Kaplan-Meier.

\section{Results \\ Success Rate of Kidney IRI Model \\ Kidney IRI was performed successfully in 182 of 186 mice with a $97.85 \%$ success rate. The deaths of 4 mice were caused by intra-abdominal hemorrhage. There was no mortality in sham operation group. There was no significant difference between the IRI group and the sham operation group in success rate $(p>0.05)$.}

\section{Effect of IRI on Scr and BUN in male mice}

In our experiments, the control animals had a Scr level of $14 \mu \mathrm{mol} / \mathrm{L}$ and a BUN value of about $6.7 \mathrm{mmol} / \mathrm{L}$. No significant differences were shown between the male group and the female group under the control condition. Following $30 \mathrm{~min}$ of ischemia, Scr and BUN increased to $34.25 \pm$ $8.62 \mu \mathrm{mol} / \mathrm{L}$ and $18.28 \pm 2.45 \mathrm{mmol} / \mathrm{L}$, within $24 \mathrm{hr}$ in male mice respectively. Male mice showed a drastic increase of Scr and BUN following $35 \mathrm{~min}-45 \mathrm{~min}$ of ischemia, to $239.2 \pm 15.82 \mu \mathrm{mol} / \mathrm{L}$ and $56.68 \pm 2.84 \mathrm{mmol} / \mathrm{L}$ (Fig. 1 ).

\section{Effect of IRI on Scr and BUN in female mice}

While the Scr and BUN values of female mice were increased after ischemia, the increase was significantly lower than that of the males (Fig. 1). The Scr and BUN were not significantly different among $0 \mathrm{~min}, 30 \mathrm{~min}, 45 \mathrm{~min}$, and 60 min groups. After $75 \mathrm{~min}$ of ischemia, serum creatinine and BUN increased to $178.5 \pm 41.00 \mu \mathrm{mol} / \mathrm{L}$ and 60.13 $\pm 1.99 \mathrm{mmol} / \mathrm{L}$ within $24 \mathrm{hr}$ in female mice respectively. These results demonstrate the resistance of female animals to IRI.

\section{Histopathologic Evaluation}

In the sham-operation groups renal tissue sections had a normal morphology (Fig. 3C, F). Histological examination of kidneys exposed to IRI showed the distinctive pattern of ischaemic renal injury, which included widespread degeneration of tubular architecture, loss of brush border, sloughing tubular epithelial cells from the basement membrane, tubular cell necrosis and intratubular cast formation especially in the outer medulla that included proximal tubule S3 segment and the thick ascending limb (mTAL), 


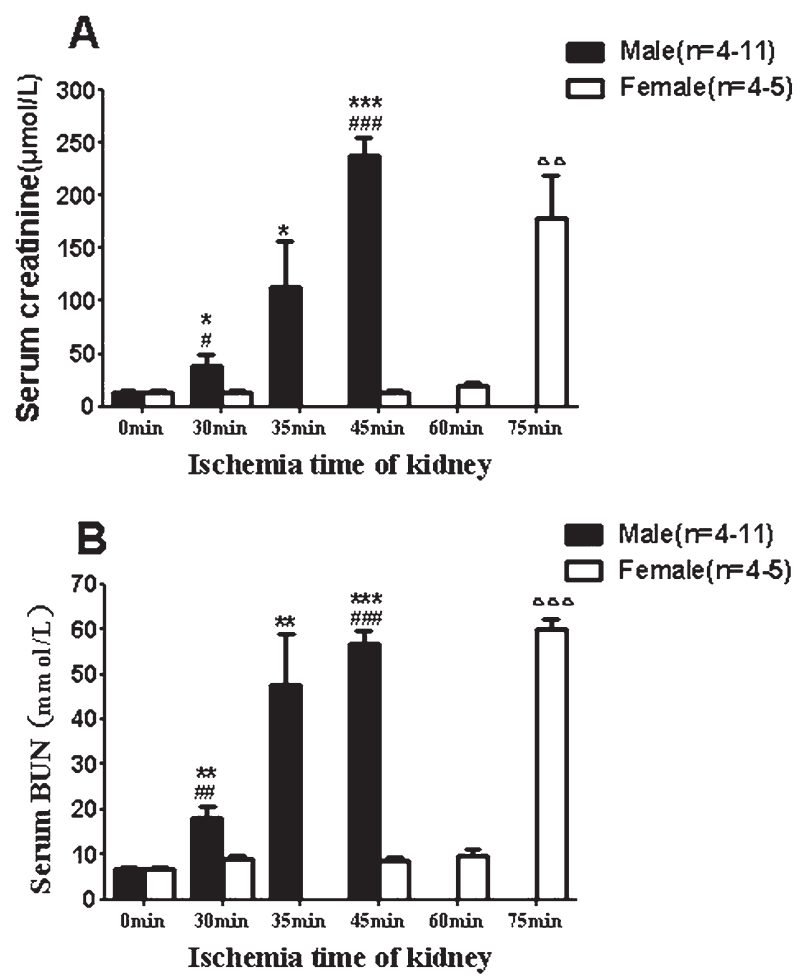

Fig. 1. Gender differences in renal function following IRI. Renal pedicles were clamped for $0 \mathrm{~min}, 30 \mathrm{~min}, 35 \mathrm{~min}$, $45 \mathrm{~min}$ in males and $0 \mathrm{~min}, 30 \mathrm{~min}, 45 \mathrm{~min}, 60 \mathrm{~min}, 75$ min in females. Blood was collected at $24 \mathrm{hr}$ after reperfusion to determine levels of Scr (A) and BUN (B). Data shown are means \pm S.E.; $* p<0.05, * * p<0.01$, *** $p<$ $0.001,{ }^{\triangle} \triangle p<0.01,{ }^{\triangle} \triangle \triangle_{p}<0.001$ versus sham operation

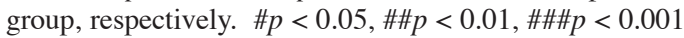
versus female mice with the same ischemia time.

two prominent sites of ischemic renal injury (Fig. 3A, B, D, E).

In 30-min, 35-min, and 45-min ischemia groups of male mice and 75-min ischemia group of females, IRI caused a significant increase in total score, when compared with the sham-operation groups $(p<0.05)$ (Fig. 2).

\section{Effect of IRI on survival of 45-min ischemia male mice}

Male mice were exposed to $45 \mathrm{~min}$ of ischemia followed by reperfusion, and survival was observed for 7 days. Most of mice were dead at 24-48 hr after reperfusion with accompanying severe lethargy and anorexia. By the end of the 7 th day, only $13.64 \%$ mice survived when submitted to 45 min of bilateral renal ischemia. However, within the same period, no mice in sham operation group were lost and their activities were normal. There was significant difference between 45-min ischemia group and sham operation group in survival $(p<0.01)$ (Fig. 4$)$.

\section{Discussion}

Native kidney surgery and kidney transplantation are associated with renal injury due to IRI, which is one of the most common causes of ARF. Experimentally, model systems of IRI from isolated renal tubules to whole animals
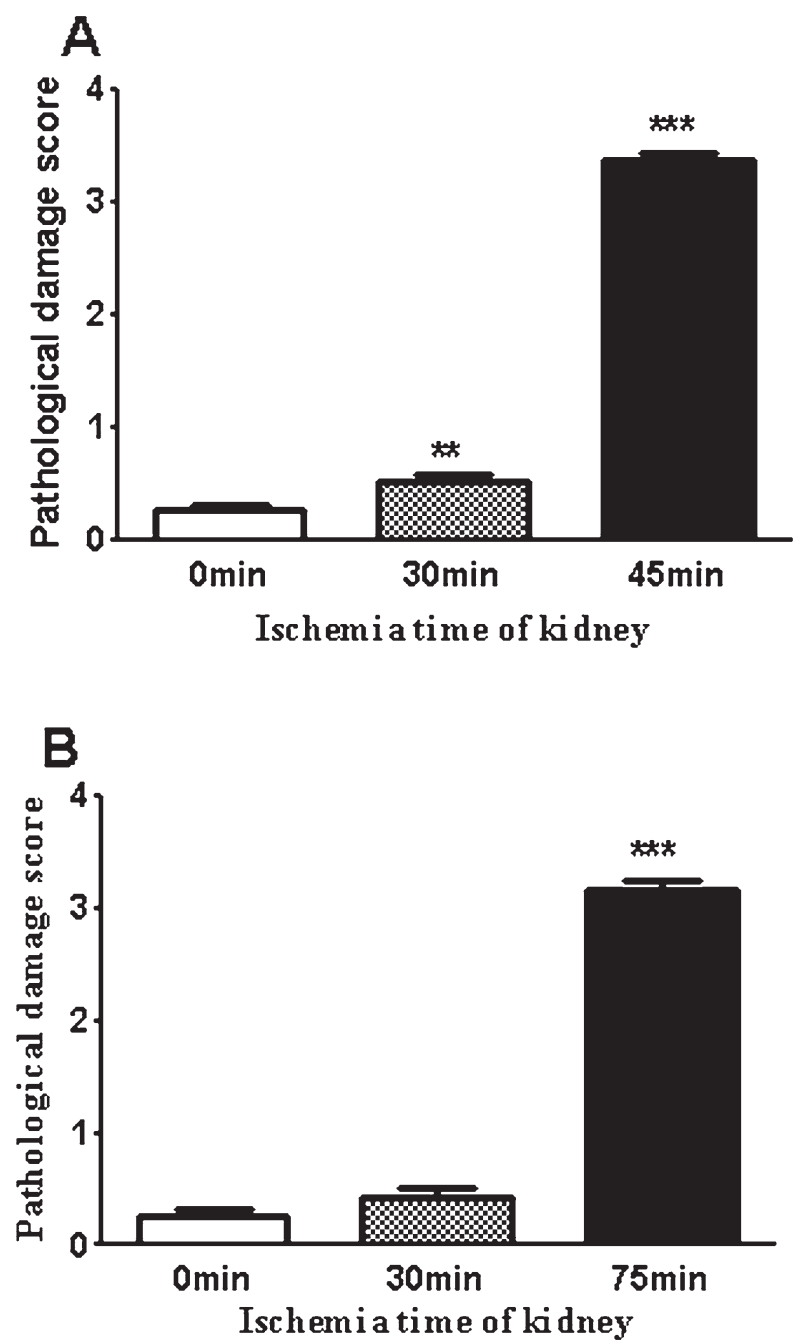

Fig. 2. Renal pathological damage score at $24 \mathrm{hr}$ after reperfusion in IRI mice.

Male mice (A) were subjected to 30 -min or 45 -min renal ischemia, and female mice (B) were subjected to 75-min renal ischemia followed by reperfusion, resulting in significantly higher pathological damage scores compared with sham operation group ( 0 min group). (**p $<0.01$, $* * * p<0.001$ versus sham operation group, respectively.) $(n=3)$.

have been explored (Lieberthal and Nigam 2000). However, due to the multifactorial features of IRI, unique systems are still desirable (Basile et al. 2004).

There are many reports on renal IRI models of big animals (Jayle et al. 2006) or rats (Chatterjee and Thiemermann 2003). However, prior to the current study, experimental models of kidney IRI in a large scale in mice with an induction of ARF were not available. In our experimental model, we have accomplished the kidney IRI models with micro-artery clamps to clamp bilateral renal arteries in $186 \mathrm{BALB} / \mathrm{c}$ mice with a $97.85 \%$ success rate. Extensive experience has accumulated as followings: (1) All animals should be fasted for 8-12 hr before the experiment, which can reduce the interference of gastrointestinal tract in the operation. (2) The depth of anesthesia should be suitable. 

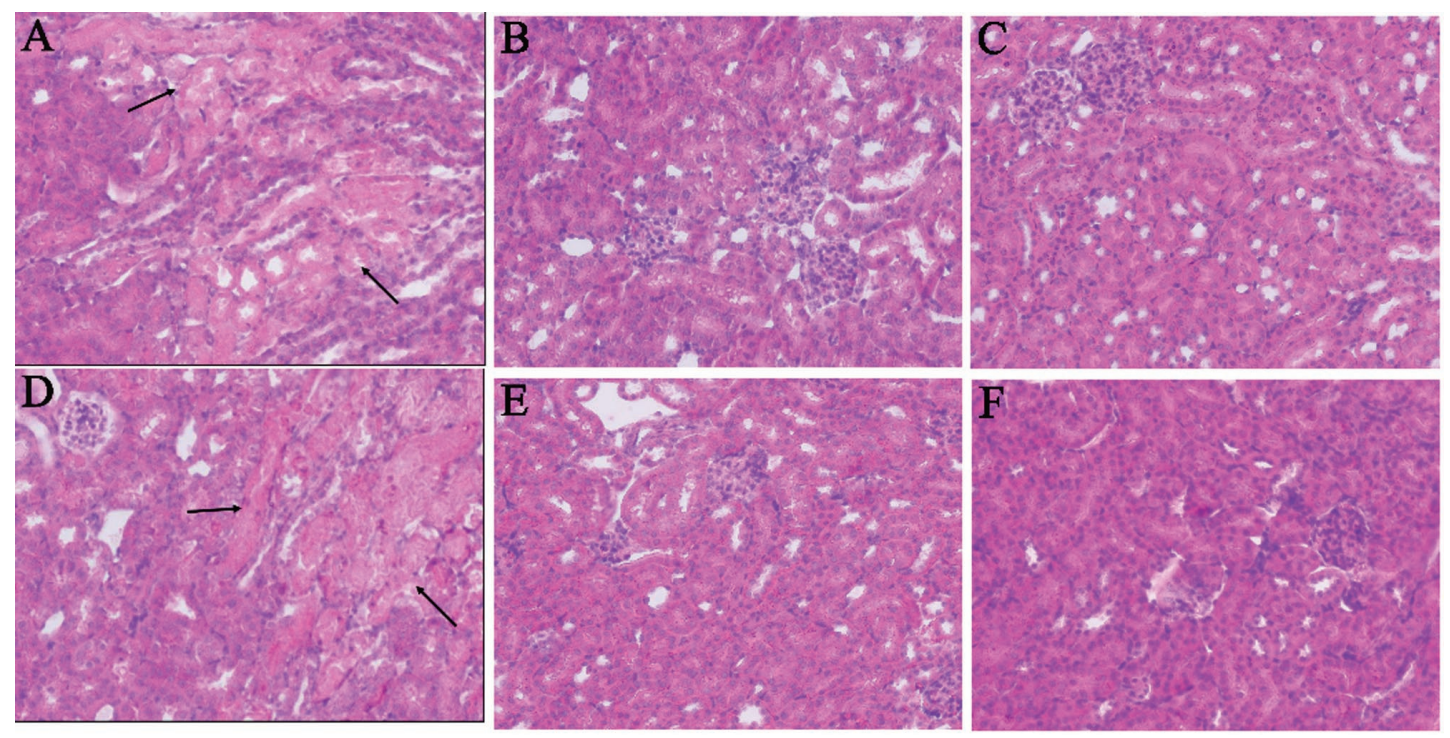

Fig. 3. Histopathological evaluation at $24 \mathrm{hr}$ after reperfusion.

Mice were subjected to different renal ischemia time followed by reperfusion. Shown are representative sections from (A) male mice with 45-min ischemia and (D) female mice with 75-min ischemia, showing widespread tubular necrosis, dilatation and vacuolization. Only marginal tissue damages are noted in the representative sections from male mice with 30-min ischemia (B) and female mice with 30-min ischemia (E). Control (non-clamped) kidneys in male (C) and female (F) show no signs of tubular necrosis. Arrows indicate tubular dilatation and necrosis. Original magnification, $\times 200$.

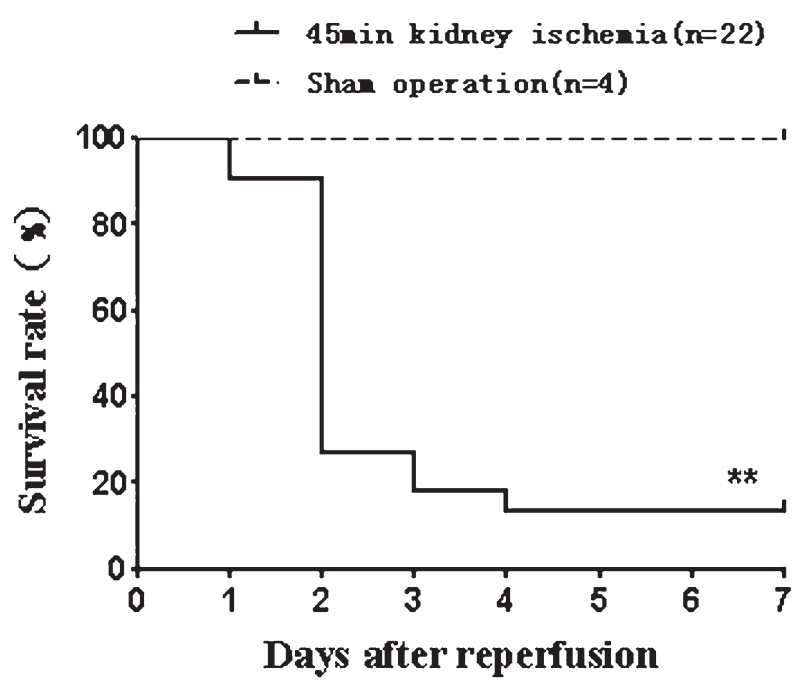

Fig. 4. The survival curve from 45 min IRI group in male mice.

The male mice were submitted to $45 \mathrm{~min}$ of bilateral renal ischemia. Survival of mice was observed over 7 days. $(* * p<0.01,45$ min group vs. sham operation group)

If the anesthetic is overdosage, it is very difficult for animals to resuscitate postoperatively. (3) During the operation, all animals should be kept well hydrated with warm saline. (4) All animals should be kept their body warm under controlled room temperature at $24 \pm 2{ }^{\circ} \mathrm{C}$. (5) Attention should be given during separating the renal pedicles. We only separated the dorsal side and inferior side of the renal pedicles bluntly. Then the micro-artery clamps can be placed correctly, with particular attention to reduce the interference around the renal pedicles. (6) The microartery clamps should be placed successfully one time; otherwise, it is very easy to induce kidney ischemic preconditioning and increase the resistance to the ischemia. (7) Do not damage the abdominal tissue and cause intraabdominal hemorrhage. Especially pay more attention to liver and spleen when clamp the renal pedicles. It is easy to lose the animals if more than $0.5 \mathrm{ml}$ total blood lost.

Our findings demonstrate that the male mice are submitted to $30 \mathrm{~min}$ of bilateral renal ischemia, then the Scr and BUN are elevated at $24 \mathrm{hr}$ after reperfusion. Moreover, the Scr and BUN are increased gradually while prolonging the ischemia time. When the male mice are submitted to $45 \mathrm{~min}$ of bilateral renal ischemia, the pathological scores show that there is widespread tubular necrosis. Survival curve shows most of mice are dead at 24-48 hr after reperfusion. But, da Silva et al. (2007) and Kale et al. (2003) reported no mice survived more than $24 \mathrm{hr}$ when submitted to 35-45 min of bilateral renal ischemia with male C57BL/6 mice and Rosa26 mice, respectively. The discrepancy with our results is probably due to mouse strain differences (Burne et al. 2000).

Our results show that in females even when the ischemic time was extended to $60 \mathrm{~min}$, there is still no an increase of Scr and BUN. When the females were submitted to 75 min of bilateral renal ischemia, there were significant changes of Scr, BUN and the pathological scores. In contrast, Park et al. (2004) reported when the ischemic time was $60 \mathrm{~min}$ in female BALB/c mice, there were significant changes of plasma creatinine. Reasons for this discrepancy were unknown, but might be due to the different subtype of $\mathrm{BALB} / \mathrm{c}$ mouse strain in the study. 
The kidneys of males are much more susceptible to IRI than those of females. Although gender differences in kidney IRI susceptibility have been characterized in rats (Fekete et al. 2004) and mice (Park et al. 2004; Wei et al. 2005), mechanistically, very limited information is available for the gender differences in kidney IRI. Szabo and colleagues suggested that estrogen might play an important role in protecting females from ischemic ARF by limiting endothelin activation (Müller et al. 2002). On the other hand, recent results by Park et al (Park et al. 2004, 2005) showed that deprivation of estrogen in female animals by ovariectomy did not affect ischemic renal injury. Instead, the presence of testosterone seemed to play a critical role in gender differences in susceptibility to ischemic ARF. They also showed that testosterone acted to inhibit NOS activation, Akt phosphorylation, and the post-ischemic increase in the ratio of extracellular signal related kinase (ERK) to c-jun N-terminal kinase (JNK) activation, leading to greater inflammatory responses.

\section{Conclusion}

In the BALB/c mice model of kidney IRI we presented in this study, the kidney of male is much more susceptible to IRI than that of female. The optimal renal ischemia time for ideal IRI model of BALB/c mice is $35-45 \mathrm{~min}$ in males and $75 \mathrm{~min}$ in females. Nevertheless, the gender differences might provide interesting models for research of the cellular and molecular basis of kidney IRI.

\section{Acknowledgments}

The authors would like to thank Dr. Xunchang Zou (Department of Medicine of Ohio State University, USA) for reviewing the manuscript.

\section{References}

Basile, D.P., Donohoe, D., Cao, X. \& Van Why, S.K. (2004) Resistance to ischemic acute renal failure in the Brown Norway rat: a new model to study cytoprotection. Kidney Int., 65, 22012211.

Baylis, C. (1994) Age-dependent glomerular damage in the rat. Dissociation between glomerular injury and both glomerular hypertension and hypertrophy. Male gender as a primary risk factor. J. Clin. Invest., 94, 1823-1829.

Burne, M.J., Haq, M., Matsuse, H., Mohapatra, S. \& Rabb, H. (2000) Genetic susceptibility to renal ischemia reperfusion injury revealed in a murine model. Transplantation, 69, 10231025.

Chatterjee, P.K. \& Thiemermann, C. (2003) An in vivo model of ischemia/reperfusion and inflammation of the kidneys of the rat. Methods Mol. Biol., 225, 223-237.

da Silva, L.B., Palma, P.V., Cury, P.M. \& Bueno, V. (2007) Evaluation of stem cell administration in a model of kidney isch- emia-reperfusion injury. Int. Immunopharmacol., 7, 16091616.

Fekete, A., Vannay, A., Vér, A., Vásárhelyi, B., Müller, V., Ouyang, N., Reusz, G., Tulassay, T. \& Szabó, A.J. (2004) Sex differences in the alterations of $\mathrm{Na}(+), \mathrm{K}(+)$-ATPase following ischaemia-reperfusion injury in the rat kidney. J. Physiol., 555 (Pt 2), 471-480.

Gobé, G., Willgoss, D., Hogg, N., Schoch, E. \& Endre, Z. (1999) Cell survival or death in renal tubular epithelium after ischemia-reperfusion injury. Kidney Int., 56, 1299-1304.

Jayle, C., Milinkevitch, S., Favreau, F., Doucet, C., Richer, J.P., Deretz, S., Mauco, G., Rabb, H. \& Hauet, T. (2006) Protective role of selectin ligand inhibition in a large animal model of kidney ischemia-reperfusion injury. Kidney Int., 69, 17491755.

Ji, H., Menini, S., Mok, K., Zheng, W., Pesce, C., Kim, J., Mulroney, S. \& Sandberg, K. (2005) Gonadal steroid regulation of renal injury in renal wrap hypertension. Am. J. Physiol. Renal Physiol., 288, F513-F520.

Kale, S., Karihaloo, A., Clark, P.R., Kashgarian, M., Krause, D.S. \& Cantley, L.G. (2003) Bone marrow stem cells contribute to repair of the ischemically injured renal tubule. J. Clin. Invest., 112, 42-49.

Lieberthal, W. \& Nigam, S.K. (2000) Acute renal failure. II. Experimental models of acute renal failure: imperfect but indispensable. Am. J. Physiol. Renal Physiol., 278, F1-F12.

Müller, V., Losonczy, G., Heemann, U., Vannay, A., Fekete, A., Reusz, G., Tulassay, T. \& Szabó, A.J. (2002) Sexual dimorphism in renal ischemia-reperfusion injury in rats: possible role of endothelin. Kidney Int., 62, 1364-1371.

Park, K.M., Cho, H.J. \& Bonventre, J.V. (2005) Orchiectomy reduces susceptibility to renal ischemic injury: a role for heat shock proteins. Biochem. Biophys. Res. Commun., 328, 312317.

Park, K.M., Kim, J.I., Ahn, Y., Bonventre, A.J. \& Bonventre, J.V. (2004) Testosterone is responsible for enhanced susceptibility of males to ischemic renal injury. J. Biol. Chem., 279, 5228252292.

Perico, N., Cattaneo, D., Sayegh, M.H. \& Remuzzi, G. (2004) Delayed graft function in kidney transplantation. Lancet, 364, 1814-1827.

Rabb, H., Mendiola, C.C., Dietz, J., Saba, S.R., Issekutz, T.B., Abanilla, F., Bonventre, J.V. \& Ramirez, G. (1994) Role of $\mathrm{CD} 11 \mathrm{a}$ and $\mathrm{CD} 11 \mathrm{~b}$ in ischemic acute renal failure in rats. Am. J. Physiol., 267(6 Pt 2), F1052-F1058.

Schlichting, C.L., Schareck, W.D. \& Weis, M. (2006) Renal ischemia-reperfusion injury: new implications of dendritic cellendothelial cell interactions. Transplant Proc., 38, 670-673.

Stringer, K.D., Komers, R., Osman, S.A., Oyama, T.T., Lindsley, J.N. \& Anderson, S. (2005) Gender hormones and the progression of experimental polycystic kidney disease. Kidney Int., 68, 1729-1739.

Tomiyoshi, Y., Sakemi, T., Aoki, S. \& Miyazono, M. (2002) Different effects of castration and estrogen administration on glomerular injury in spontaneously hyperglycemic Otsuka LongEvans Tokushima Fatty (OLETF) rats. Nephron., 92, 860-867.

Wei, Q., Wang, M.H. \& Dong, Z. (2005) Differential gender differences in ischemic and nephrotoxic acute renal failure. Am.J. Nephrol., 25, 491-499. 